\title{
Highlights from the Pierre Auger Observatory
}

\author{
Piera Luisa Ghia ${ }^{* a}$ for the Pierre Auger Collaboration ${ }^{b}$ \\ ${ }^{a}$ Laboratoire de Physique Nucléaire et de Hautes Energies (LPNHE), CNRS-IN2P3, Université \\ Pierre et Marie Curie et Université Paris Diderot, Paris, France \\ ${ }^{b}$ For the complete author list, see http://www.auger.org/archive/authors_2015_06.html \\ E-mail: piera.ghia@lpnhe.in2p3.fr
}

\begin{abstract}
The Pierre Auger Observatory, located near the town of Malargüe, Argentina, has been detecting ultra-high energy cosmic rays for more than ten years. An essential feature of the $3000 \mathrm{~km}^{2} \mathrm{Ob}-$ servatory is its hybrid design: cosmic rays above $10^{17} \mathrm{eV}$ are detected through the observation of the associated air showers with different and complementary techniques, from surface detector arrays and fluorescence telescopes to radio antennas. The analyses of the multi-detector data have enabled high-statistics and high-precision studies of the energy spectrum, mass composition and distribution of arrival directions of ultra-high energy cosmic rays (UHECRs), the investigation of whose origin being a primary objective of the Observatory. The resulting picture, calling into question the perception of UHECRs at the time of the conception of the Observatory in the early 1990s, has led to a plan for an upgrade of the Observatory. The variety of the instruments and analyses has also allowed us to touch upon other aspects than just the astrophysics of UHECRs: information of relevance to high-energy particle interactions as well as to solar and heliospheric phenomena is part of the science harvest from 10 years of operation presented at the 34th International Cosmic Ray Conference.
\end{abstract}

The 34th International Cosmic Ray Conference,

30 July- 6 August, 2015

The Hague, The Netherlands

\footnotetext{
* Speaker.
} 


\section{Introduction}

The Pierre Auger Observatory [1] is the fruit of the efforts of a 450-people international collaboration - including 16 countries and 68 institutions - with the pivotal help of an on-site team of physicists, engineers and technicians. Conceived nearly 25 years ago as a hybrid system of a giant surface array of particle detectors coupled with air fluorescence telescopes, its objectives have been to measure, with high statistics and precision, the energy, mass and arrival directions of cosmic rays above $10^{19} \mathrm{eV}$, i.e., in the region of the so-called GZK cutoff [2], at that time scarcely explored. Ten ICRCs after the first presentation of the project to the community [3], the 28 contributions presented at the 34th ICRC offer the opportunity to take stock of the status of the Observatory and show that the reach of the results, obtained in more than 10 years of operation, is actually much wider than what was originally envisioned. It is wider in terms of the energy range explored, extending now down to $10^{17} \mathrm{eV}$, thanks to enhancements of the baseline design, always keeping with the original hybrid-detection strategy. The ensemble of instruments (outlined in Section 2) has made possible the study of ultra-high energy cosmic rays over more than three decades in energy - as illustrated in Section 3 - thus also including the region where the transition from a Galactic to an extragalactic origin of cosmic rays is expected, in addition to that of the GZK cutoff. The reach of the results is wider in scope too, touching upon aspects other than the physics of cosmic rays at ultra-high energies. As shown in Section 4, creative and sophisticated analyses of data collected by the different detectors have been used to probe high-energy interactions of particles beyond the reach of the LHC, even searching for exotic particles. The versatility of the Auger instruments has also granted access to the study of the flux of cosmic rays at $\mathrm{GeV}$ energies, relevant to solar and heliospheric phenomena. Understanding the precise origin of the highest energy cosmic rays still remains the not fully realized objective of the Observatory. The mostly unexpected picture emerging from the analyses of 10 years of data has led the Collaboration to conceive a plan for an upgrade of the Observatory, whose motivations and design are outlined in Section 5. ${ }^{1}$

\section{Auger instruments}

The most prominent characteristic of the Auger Observatory, in operation since 2004, is its capability of observing extensive air showers (EAS) with different instruments, that complement each other in terms of measurement of shower components and of the energy range accessed.

1600 water-Cherenkov detectors (WCDs), spaced by $1500 \mathrm{~m}$, form the $3000 \mathrm{~km}^{2}$ surface detector array (SD-1500 m). After the completion of the latter in 2008, a smaller, denser SD of $23.5 \mathrm{~km}^{2}$ area comprised of $61 \mathrm{WCDs}$, was added, nested within the former one. Due to its finer spacing of $750 \mathrm{~m}$ (SD-750 m), it extends the energy range of the SD-1500 m (fully efficient at energies above $3 \times 10^{18} \mathrm{eV}$ ) down to $3 \times 10^{17} \mathrm{eV}$. The SDs continuously sample the shower particles that reach the ground. The signals registered in the WCDs, due to both the electromagnetic and muonic components of EAS, are used to determine the shower geometry (core position and arrival

\footnotetext{
${ }^{1}$ For the sake of higlighting as much as possible the variety of the measurements and their implications, the author perforce had to sacrifice most details of the analyses in this report. An interested reader is invited to refer to the individual contributions whose ensemble has been made available on the arXiv $[4,5,6]$. To facilitate their identification, the different contributions higlighted here are referred to using their presenter's name.
} 
direction) and an estimator of the primary energy. The two SDs are overlooked by a fluorescence detector (FD) consisting of five units. Four of them, including 6 telescopes each, overlook the SD-1500 m array. Each telescope has a field of view (FOV) of $30^{\circ} \times 30^{\circ}$ in azimuth and elevation, with a minimum elevation of $1.5^{\circ}$ above the horizon. The fifth one (named HEAT, High Elevation Auger Telescope), overlooking the SD-750 m array with three telescopes whose FOV in elevation goes from $30^{\circ}$ to $60^{\circ}$, extends downwards the FD energy range by one decade, from a threshold of $10^{18} \mathrm{eV}$ to $10^{17} \mathrm{eV}$. In all 27 telescopes, the light is focused by spherical mirrors on cameras built of 440 photomultipliers (PMTs). The FD measures, with a 15\% duty cycle, the longitudinal profile of the shower via the amount of nitrogen fluorescence produced along its path, dominantly by the electromagnetic component. The integral of the longitudinal profile is used to determine the energy of the primary, while the observed depth at which the shower reaches its maximum size is indicative of the mass. Given the relevance of the atmosphere for the FD, the Observatory includes also several devices to monitor atmospheric parameters, in particular clouds and aerosols that influence both the production of fluorescence light and its attenuation. Four lidars plus the Central Laser Facility (CLF) and the eXtreme Laser Facility are pivotal to that.

With all these instruments smoothly taking data, the activity is now focused on the monitoring of their performance as well as on a deeper understanding of their response. This is of great relevance in the present analysis phase, where long-term data and more and more refined analyses are exploited. Three contributions are emblematic of this kind of activity. The resistive plate chamber hodoscope installed at one of the WCDs, described by P. Assis, serves to fine-tune the detector simulations, extensively used in sophisticated exploitations of the signals detected in the WCDs. Monitoring the long-term stability of FD data is crucial to the analysis: the 500-million-record database described by G. Salina, which stores calibration data for the 12000 PMTs of the FD in 10 years of operation, is vital to that. In connection to the quality of FD data, the inclusion of a Raman LIDAR receiver in the recently upgraded CLF, illustrated by C. Medina, adds further redundancy to the monitoring of the atmosphere, providing an independent measurement of the vertical aerosol optical depth. In the context of detector activity, it is worth mentioning the newly started instrumental program in collaboration with the Telescope Array. Presented by R. Takeishi, the deployment of 8 Auger WCDs at the Telescope Array site, meant to compare data at detector- and event-level, is part of a wider inter-collaboration program that regards also aspects of the analyses of UHECR data, as shown in the following sections.

The construction activity has in fact not ceased at the Observatory, as the Auger Engineering Radio Array (AERA) was being deployed and completed at the beginning of 2015, as illustrated by J. Schulz. Extending the SD+FD hybrid design to include EAS radio detection, it consists of a graded array (from $144 \mathrm{~m}$ to $750 \mathrm{~m}$ spacing) over a $17 \mathrm{~km}^{2}$ area, with 153 radio detectors operating in the $30-80 \mathrm{MHz}$ band. Targeting the detection of cosmic rays above $10^{17} \mathrm{eV}$, it is located within the SD-750 m array and close to HEAT. More than 5500 showers have already been recorded by AERA in coincidence with the SD, and about 300 also with the FD. These multi-hybrid events have been shown to be powerful in studying the resolutions of AERA in reconstructing the EAS arrival direction and the energy, as well as to identify mass-sensitive radio parameters. As radio detection requires the monitoring of the environmental electric field, E-field mills and lightning detection devices have been installed to assist AERA, as explained in J. Rautenberg's contribution.

The power of the hybrid detection strategy will be manifest in most studies described in the 

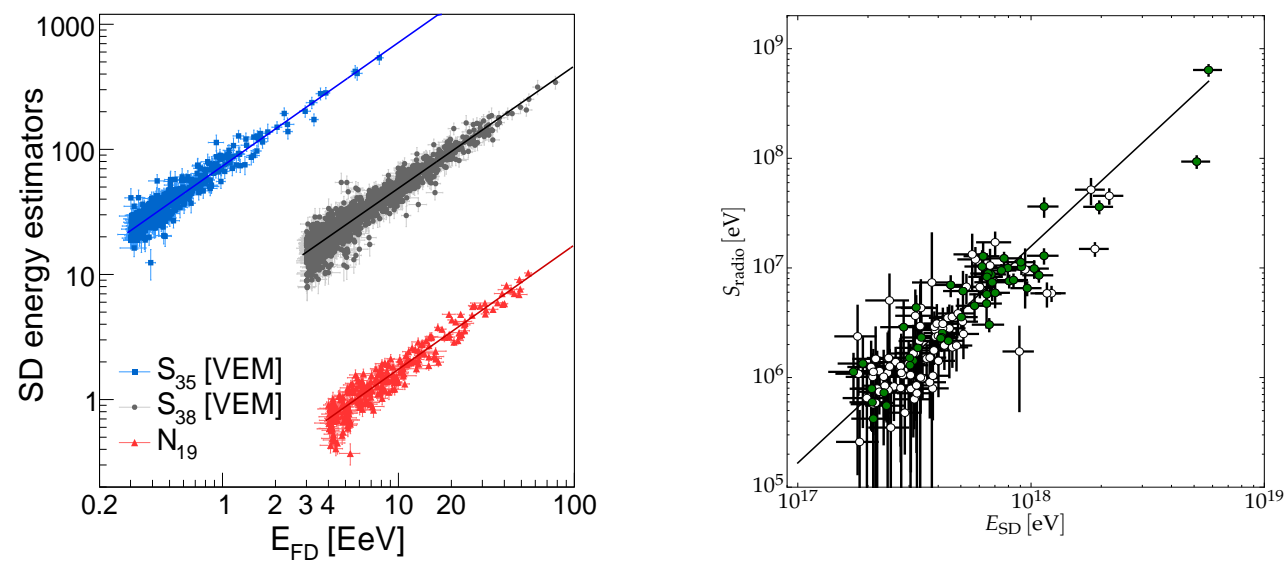

Figure 1: Left: Correlation between the SD energy estimators (see text) and the FD energy. Right: Correlation between the radio energy estimator $S_{\text {radio }}$ and the energy measured with the SD.

next sections. Its strength is already apparent however at the level of cosmic-ray reconstruction, in that the FD provides the Observatory with a common energy scale. Thanks to the FD capability of determining the primary energy with a nearly calorimetric measurement, the energy determination, besides being common, is also free of cascade simulation uncertainties and of the modelling of hadronic interactions behind them. A high-quality subset of hybrid events (i.e., detected simultaneously by the FD and at least $1 \mathrm{WCD}$ of the SD) is used to calibrate the SD energy estimators with the energies measured with the FD. The calibrations, updated to include $20 \%$ more hybrid events in I. Valiño's contribution, are shown in Fig. 1, left panel. $S_{35}$ and $S_{38}$ are the energy estimators for the so-called vertical showers $\left(\theta<60^{\circ}\right)$ recorded by SD-750 m and SD-1500 m, respectively: they are the signals at $450 \mathrm{~m}$ and $1000 \mathrm{~m}$, respectively, corrected for atmospheric absorption. $N_{19}$ is the energy estimator for the so-called horizontal events $\left(60^{\circ}<\theta<80^{\circ}\right)$ : it is a proxy for the muon content that is directly related to the primary energy (see also Section 4$)$. The three correlations are well described by simple power-law functions $E_{\mathrm{FD}}=A(\hat{S})^{B}$ with $\hat{S}=S_{38}, S_{35}$ or $N_{19}$. With a similar procedure, the FD energy scale has been propagated to AERA data, as presented by C. Glaser. This, due to statistical limitations, has been performed against SD data, by using hybrid SD-radio events. $S_{\text {radio }}$, i.e., the energy radiated in the form of radio emission in the 30-80 $\mathrm{MHz}$ range, is derived from the conversion of the measured electric field in all radio stations into energy fluence. The latter are fitted with a two-dimensional lateral distribution function and integrated over the shower plane to determine $S_{\text {radio }}$. The calibration of this radiation energy against the cosmic-ray energy is shown in Fig. 1, right panel: $S_{\text {radio }}$ scales quadratically with the shower energy, as expected for coherent radio emission. Note that this represents the first measurement of the energy in the radio signal of EAS. As a result of the above-described calibrations, the SDs and AERA share the uncertainty of the FD energy scale, of $14 \%$ (16\%) above (below) $10^{18} \mathrm{eV}$ [7].

\section{Ultra-high energy cosmic ray science}

The measurement of the energy spectrum of UHECRs, the inference on their mass composition and the analysis of their arrival directions bring different information, complementary and supple- 

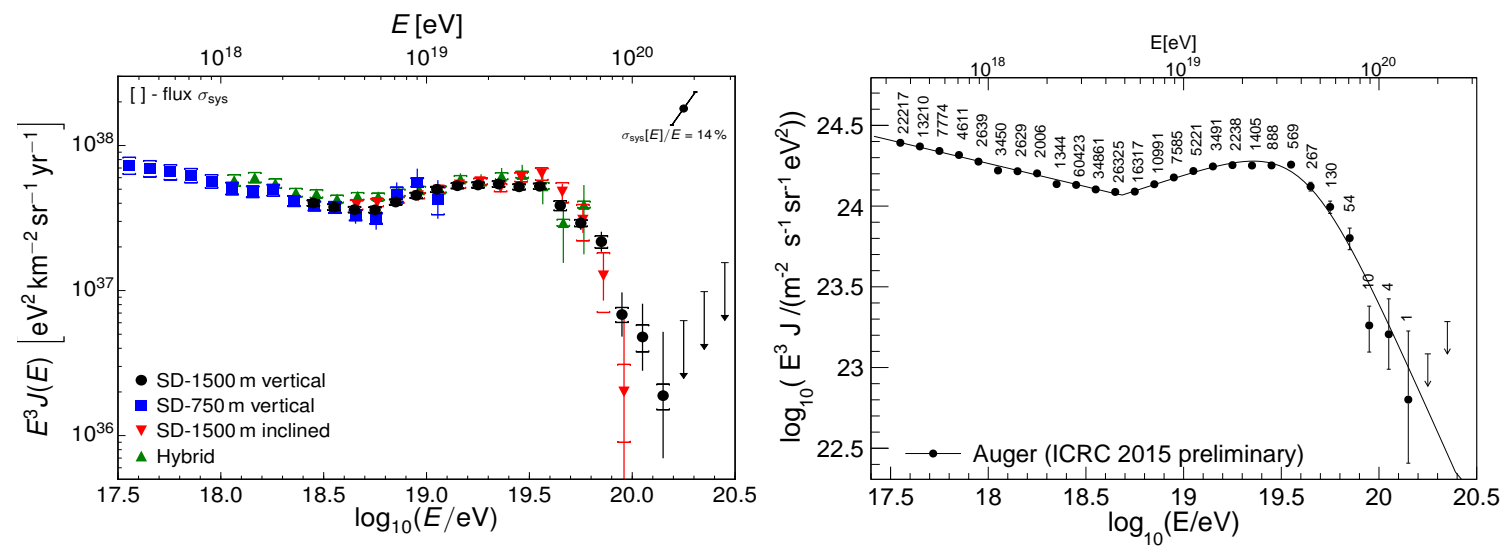

Figure 2: Left: The four energy spectra derived from SD and hybrid data. Right: The combined energy spectrum, fitted with a flux model (see text). As in the left panel, only statistical uncertainties are shown and the upper limits correspond to $84 \%$ confidence level (C.L.).

mentary to one another, with respect to their origin. In this section the suite of reports presented by the Auger Collaboration on these topics is highlighted all together, in an attempt to underline their reciprocal relation in deriving inferences on UHECRs.

The measurement of the flux of UHECRs has been one of the first results of Auger [8]. Two spectral features have been established beyond doubt: the hardening in the spectrum at about $5 \times 10^{18} \mathrm{eV}$ (the so-called ankle), and a strong suppression of the flux at the highest energies, starting at about $4 \times 10^{19} \mathrm{eV}$. The all-particle flux of cosmic rays presented by I. Valiño is an update of this measurement, based on an exposure now larger than $50000 \mathrm{~km}^{2} \mathrm{sr} \mathrm{yr}$ and on $\sim 200000$ events. Combining four independent spectra (see Fig. 2, left) from the two different SDs (and two data sets from the SD-1500 m, vertical and horizontal events) and from hybrid events, the measurement is emblematic of the power of using multiple detectors. Data from the SD-750 m allow for the determination of the energy spectrum down to $10^{17} \mathrm{eV}$. The SD-1500 m vertical data are crucial above the energy of full trigger efficiency of $3 \times 10^{18}$ up to the highest energies, with horizontal events contributing above $4 \times 10^{18} \mathrm{eV}$ and providing an independent measurement in this energy range. Hybrid data bridge those from the two SDs, between $10^{18} \mathrm{eV}$ and $10^{19.6} \mathrm{eV}$. The four spectra, in agreement within uncertainties, are combined into a unique one shown in Fig. 2, right panel, taking into account the systematics of the individual measurements. The evident features are quantified by fitting a model that describes the spectrum with two power laws around the ankle, the second of which includes a smooth suppression at the highest energies. The ankle is found to be at $E_{\text {ankle }}=(4.8 \pm 0.1 \pm 0.8) \times 10^{18} \mathrm{eV}$. The spectral slope below the ankle is $\gamma_{1}=3.29 \pm 0.02 \pm 0.05$, and above the ankle is $\gamma_{2}=2.60 \pm 0.02 \pm 0.10$. The energy at which the differential flux falls to one-half of the value of the power-law extrapolation is $E_{\mathrm{s}}=(42.1 \pm 1.7 \pm 7.6) \times 10^{18} \mathrm{eV}$. In each case the precision of the measurements is now limited by the systematic uncertainty.

The origin of the very precisely determined features in the all-particle spectrum has been addressed by the Collaboration in parallel, likewise for many years [9], through the measurement of the depth of the shower maximum, $X_{\max }$, one of the most robust mass-sensitive EAS observables. The measurement, relying on hybrid data, has been recently updated in [10]: it has also been presented by A. Porcelli, and extended for the first time to cover the whole energy range of 

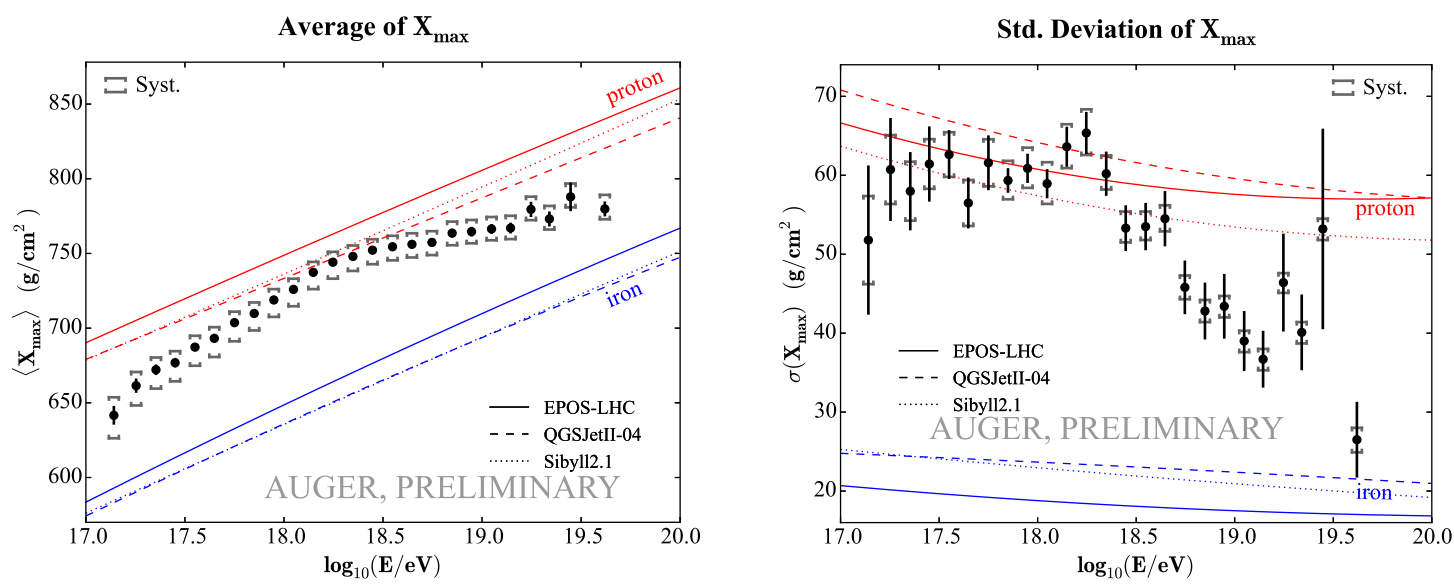

Figure 3: The mean (left) and the standard deviation (right) of the measured $X_{\max }$ distributions as a function of energy compared to air-shower simulations for proton and iron primaries.

the all-particle flux, down to $10^{17} \mathrm{eV}$, thanks to the inclusion of data from the HEAT telescopes. More than 18000 events collected by the standard FD telescopes (FD data) above $10^{17.8} \mathrm{eV}$, have been supplemented by $\approx 5500$ events collected with HEAT in coincidence with the closest FD, Coihueco (so-called HeCo data). By using for HeCo data the same selection and analysis strategy as for FD data [10], the first two moments of the $X_{\max }$ distributions, $\left\langle X_{\max }\right\rangle$ and $\sigma\left(X_{\max }\right)$, are estimated from $10^{17} \mathrm{eV}$ to $10^{18.3} \mathrm{eV}$. In agreement within uncertainties with those derived from FD data in the common energy range (from $10^{17.8} \mathrm{eV}$ to $10^{18.3} \mathrm{eV}$ ), they are combined into the resulting $\left\langle X_{\max }\right\rangle$ and $\sigma\left(X_{\max }\right)$ shown in Fig. 3, left and right, respectively, as a function of energy. Data are compared to simulations, for proton and iron primaries, performed using three hadronic interaction models that were either tuned to recent LHC data (QGSJetII-04 [11], EPOS-LHC [12]) or found in good agreement with LHC measurements (Sibyll2.1 [13]). Between $10^{17.0}$ and $10^{18.3} \mathrm{eV}$, $\left\langle X_{\max }\right\rangle$ increases by around $85 \mathrm{~g} \mathrm{~cm}^{-2}$ per decade of energy; above $\approx 10^{18.3} \mathrm{eV}$, the rate of change of $\left\langle X_{\max }\right\rangle$ becomes significantly smaller $\left(\sim 26 \mathrm{~g} \mathrm{~cm}^{-2}\right.$ per decade). These two values, consistent with those found with FD data alone [10], allow us to extend the inferences on the evolution of the average mass composition down to $10^{17.0} \mathrm{eV}$. As the first value is larger than the one expected for a constant mass composition ( $\sim 60 \mathrm{~g} \mathrm{~cm}^{-2}$ per decade), it indicates that the mean primary mass is becoming lighter all the way from $10^{17.0}$ to $\approx 10^{18.3} \mathrm{eV}$. Above this energy, the trend inverts and the composition grows heavier. The fluctuations of $X_{\max }$ start to decrease above the same energy $\approx 10^{18.3} \mathrm{eV}$, being rather constant below. The conversion of the first two moments of the $X_{\max }$ distributions to the mean value of $\ln \mathrm{A}$ and its variance $\sigma^{2}(\ln \mathrm{A})$, presented also in Porcelli's contribution, confirms, in a more quantitative way, such an evolution of the average composition, consistently for the considered models.

From the energy evolution of the first two moments of the $X_{\max }$ and $\ln$ A distributions, the composition appears to be lightest around half the energy where the ankle is observed in the spectrum. Also, the inference on mass composition, made by using not only the moments of the $X_{\max }$ distributions but also the shape [14], is that a substantial fraction of protons $(\sim 60 \%$, consistently for different models) is present around the ankle. The question of whether the composition consists entirely of protons is thus natural and is of interest in shedding light on the origin of the ankle, as one 

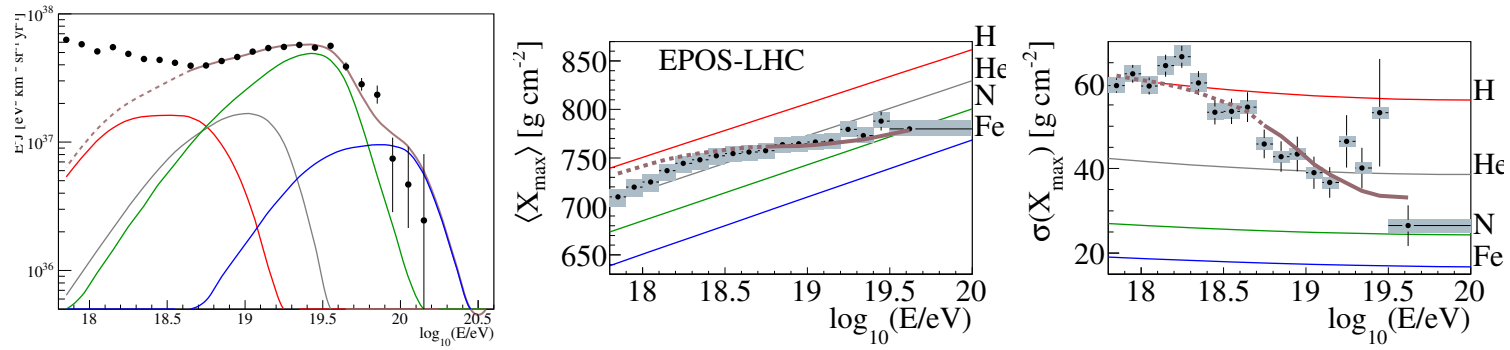

Figure 4: Left: Energy spectrum of UHECRs at Earth with the best-fit elemental contributions (see text). Centre and right: Resulting average and standard deviation of the $X_{\max }$ distributions (assuming EPOS-LHC for the interactions) for the model prediction (brown), pure ${ }^{1} \mathrm{H}$ (red), ${ }^{4} \mathrm{He}$ (grey), ${ }^{14} \mathrm{~N}$ (green) and ${ }^{56} \mathrm{Fe}$ (blue). Only the energy range where the brown lines are solid is included in the fit.

possibility [15] is that this is a "dip" due to energy loss of extragalactic protons to electron-positron pair production during propagation in the cosmic microwave background (CMB). This question has been addressed in a novel study presented by A. Yushkov, which exploits once again the power of the hybrid nature of the Observatory. The analysis is based on the concept that the correlation between $X_{\max }$ and the number of muons $\mathrm{N}_{\mu}$ is sensitive to whether the primary beam is composed of several or just one nuclear species. As $\mathrm{N}_{\mu}$ can be estimated only in a very limited sample of data (as shown in Section 4), the study relies on the signal measured in the SD detector at $1000 \mathrm{~m}$ from the EAS core, $\mathrm{S}(1000)$. This is dominated by the muonic component indeed. Yushkov showed that the correlation between $\mathrm{S}(1000)$ and $X_{\max }$ is close to zero or positive for pure primary beams, consistently for the three hadronic interaction models considered, EPOS-LHC, QGSJetII-04 and Sibyll 2.1. In turn, for compositions with a large spread of masses $(\sigma(\ln \mathrm{A}) \gtrsim 1)$, and for all models, the correlation becomes negative (see also [16]). For 1376 hybrid events at energies between $10^{18.5}$ $\mathrm{eV}$ and $10^{19.0} \mathrm{eV}$, the correlation $r$ is found to be significantly negative, $r=-0.125 \pm 0.024$ (stat). This result, robust with respect to models, points to a mixed primary composition around the ankle, disfavoring the "dip" model.

While $X_{\max }$ data, and their interpretation in terms of mass, cover the region of the ankle well, they do not extend into the region of flux suppression, due to the intrinsically limited duty cycle of the FD. However, an attempt to understand the origin of this feature has been made by simultaneously fitting both the spectrum and the evolution of $X_{\max }$ data towards the highest energies, above $10^{18.7} \mathrm{eV}$. The astrophysical model, presented by A. di Matteo, assumes identical sources, homogeneously distributed in a comoving volume, injecting ${ }^{1} \mathrm{H},{ }^{4} \mathrm{He},{ }^{14} \mathrm{~N}$ and ${ }^{56} \mathrm{Fe}$ nuclei. The spectrum at the source is taken as a power law with a rigidity-dependent broken exponential cutoff. Cosmic rays are propagated from the sources to the observer using the SimProp code [17] . The free parameters of the fit are the normalization of the flux at the source, $J_{0}$, its spectral index, $\gamma$ and its cutoff rigidity, $R_{\text {cut }}$, as well as the elemental fractions at the source. The simulated spectra and the mean and variance of the simulated $X_{\max }$ distributions corresponding to the best fit are shown in Fig. 4, left, centre and right panels, respectively, together with data. The spectrum is best fitted by a succession of cutoffs of the different groups of elements, with $R_{\text {cut }}=10^{18.67 \pm 0.03} \mathrm{~V}$, thus pointing to the flux at Earth being partly limited by the maximum energy at the source. The best fit returns $\gamma=0.94_{-0.10}^{+0.09}$, suggesting a very hard source spectrum, and an injection of mostly intermediatemass nuclei, with very few protons or iron nuclei. It has to be noted that the fit also finds a second 

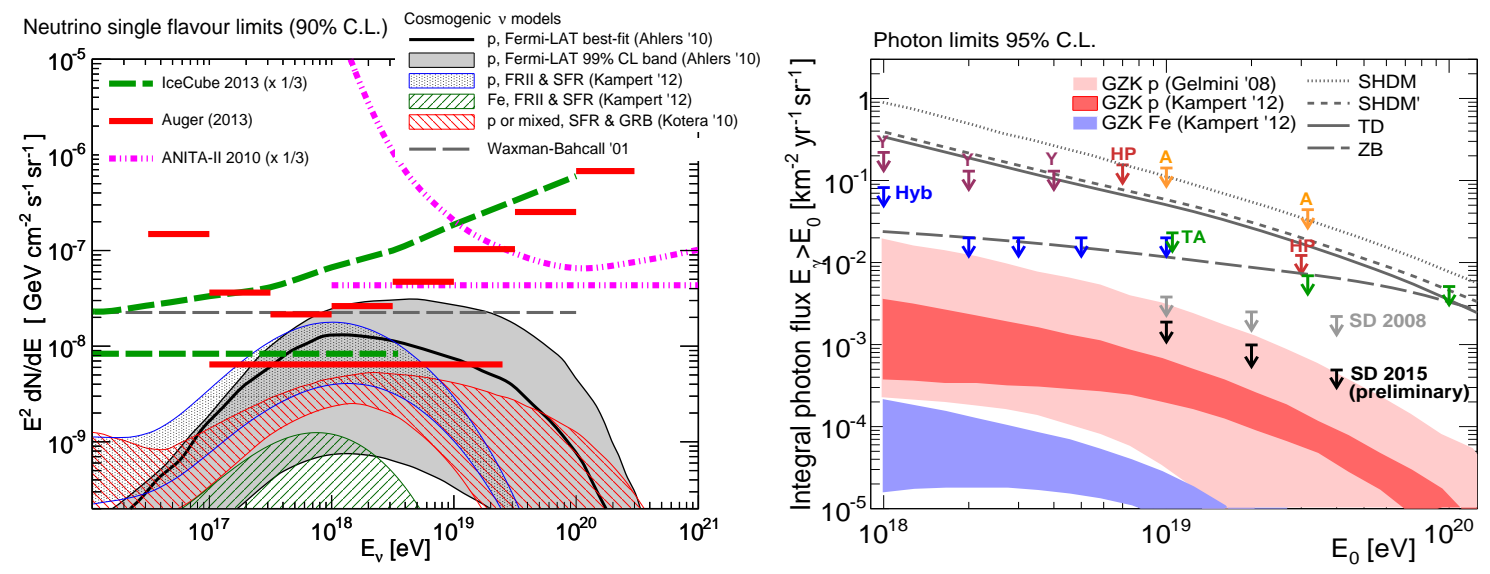

Figure 5: Left: Upper limits (red lines) to the diffuse flux of UHE neutrinos at the 90\% C.L. in integrated (horizontal lines) and differential forms. Limits are compared with cosmogenic neutrino models, the Waxman-Bahcall bound, and limits from IceCube and ANITA. All neutrino limits and fluxes are converted to single flavor. Right: Upper limits at the 95\% C.L. to the diffuse flux of UHE photons shown together with results from other experiments and predictions from several top-down and cosmogenic photon models. For the detailed references, see Bleve's report.

local minimum, with $\gamma \approx 2$ and a larger maximum rigidity, more in line with standard models of cosmic-ray acceleration. While the spectrum is fitted well in this case too, wider distributions of UHECR masses than observed in the data are in turn predicted at each energy, showing how crucial the measures of mass composition are to resolve the origin of the observed flux suppression.

Another knob to probe the origin of the flux suppression is the search for UHE photons and neutrinos. Interactions between UHECRs and photons of the CMB lead to emission of cosmogenic neutrinos and photons, whose flux is also dependent on the mass of UHECRs, being suppressed in the case of heavy primaries. The updated status of such searches was illustrated by C. Bleve, exploiting the 10-year SD data set. The two searches have much in common, besides their inferences. Neutrino- and photon-showers can be identified through the time structure of the signals in the WCDs: as the EAS electromagnetic component is larger than in hadronic showers, a broader time structure is expected. As for neutrinos, this fact is exploited for events at large zenith angles $\left(\theta>60^{\circ}\right)$. While for nuclear primaries the electromagnetic component gets absorbed due to the large thickness of traversed atmosphere, for neutrino showers, which can initiate very deep in the atmosphere, it is abundant. Downward-going neutrinos of all flavors are searched for at zenith angles $\theta>60^{\circ}$ and upward-going tau neutrinos at $\theta>90^{\circ}$. Photons are searched for in data with zenith range $30^{\circ}-60^{\circ}$ : the lower cut ensures that photon showers at these energies are fully developed at the depth of the SD. The selection criteria are additionally based on the lateral distribution of particles, steeper than in nuclear showers. Another common feature of the two searches is that the cosmic-ray background is not simulated, but determined through a fraction of data used as a "training" sample. After application of the selection criteria to data, no neutrino event survives the selection, while 4 events survive the photon search, compatible with background expectations. The derived upper limits to their fluxes, assuming a differential flux $d N(E)=k \cdot E^{-2}$, are shown in Fig. 5, left and right for neutrinos and photons, respectively. As for neutrinos, it is the first demonstration, made with a shower array, of a limit that is below the Waxman-Bahcall bound [18]. Both neutrino and photon limits reach predictions (only the more optimistic ones for photons) in the case 

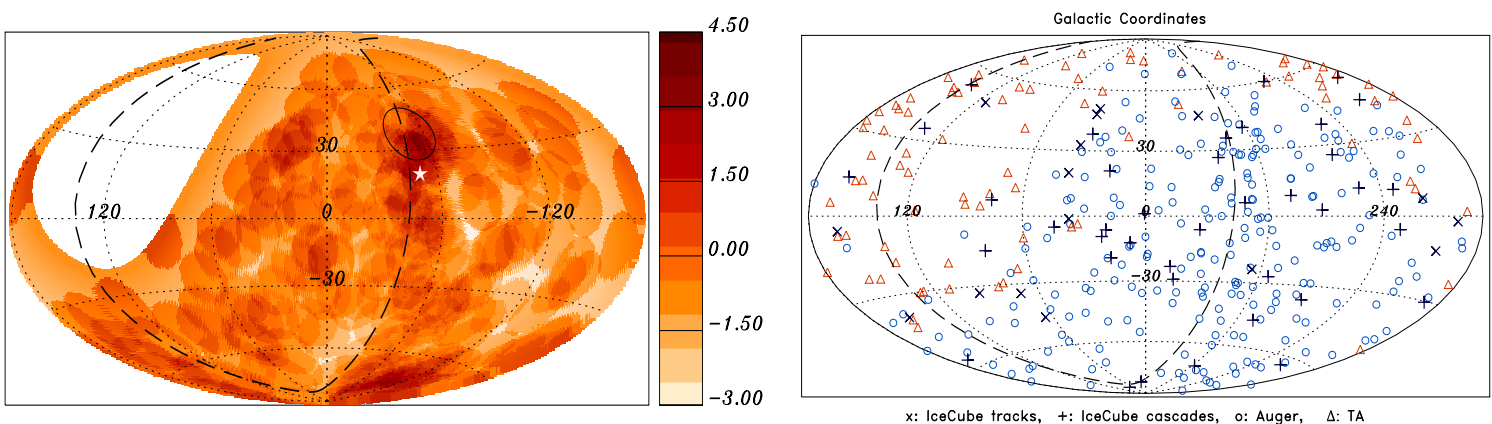

Figure 6: Left: Map in Galactic coordinates of the significances of excesses in $12^{\circ}$-radius windows for the events with $E \geq 54 \mathrm{EeV}$. The super-Galactic plane (dashed line) and Cen A (white star) are indicated. Right: Map in Galactic coordinates showing the arrival directions of the IceCube cascades (plus signs) and high-energy tracks (crosses), and of the UHECRs detected by Auger (circles) and Telescope Array (triangles). The dashed line indicates the super-Galactic plane.

of a pure proton composition at the sources. Neutrino limits in particular disfavor strong-evolution sources producing protons only. The photon limits are also compared to the fluxes expected in the case of top-down models of UHECR production, e.g., from the decay of super-heavy relic particles from the early Universe: they appear to be clearly disfavored, suggesting acceleration mechanisms for the sources of UHECRs.

If the sources are astrophysical, they should be relatively close, due to the energy losses of UHECRs in the propagation through the CMB. The distribution of the arrival directions of UHECRs above $40 \mathrm{EeV}$ might thus be anisotropic, mirroring the inhomogeneous distribution of the nearby $(\mathrm{O}(100 \mathrm{Mpc}))$ extragalactic matter, provided that the cosmic-ray charge is low. For protons above that energy the angular deflections caused by intervening magnetic fields are indeed expected to be of the order of a few degrees, while they are $\mathrm{Z}$ times larger in the case of nuclei with atomic number $Z$. In this respect, the search for anisotropy at small and intermediate angular scale at the highest energies is complementary to the spectrum and mass measurements, and is the subject of the contribution presented by J. Aublin, summarizing the work of [19]. It exploits 602 events with energy above $40 \mathrm{EeV}$, collected in 10 years of data, corresponding to an exposure ${ }^{2}$ of 66452 $\mathrm{km}^{2} \mathrm{sr} \mathrm{yr}$ and a field of view from $-90^{\circ}$ to $+45^{\circ}$ in declination. The dataset is subjected to various tests to search for anisotropies, at different energy thresholds, up to $80 \mathrm{EeV}$, and within different angular windows, between $1^{\circ}$ and $30^{\circ}$. They consider, on the one hand, the study of "intrinsic" anisotropies through the search for excesses of events over the exposed sky and the analysis of the autocorrelation of arrival directions. On the other hand, correlations are searched for with known astrophysical structures, such as the Galactic and super-Galactic planes, the Galactic centre, and with objects that are considered plausible candidates for UHECR sources. Flux-limited catalogs of galaxies (2MRS [20]), of AGNs observed in X-rays (Swift BAT-70 [21]) and of radio galaxies with jets [22] are considered. The cross-correlation with the three sets of objects is done by selecting them up to different maximum distances, i.e., based on their apparent luminosity. In the case of the AGNs in the Swift and radio-galaxy catalogs an additional scan is performed on the measured in-

\footnotetext{
${ }^{2}$ Given the large multiplicity of triggered detectors (on average, more than 14 ) at the energies considered, less stringent fiducial criteria (yielding a larger exposure) are used than for the measurement of the flux.
} 

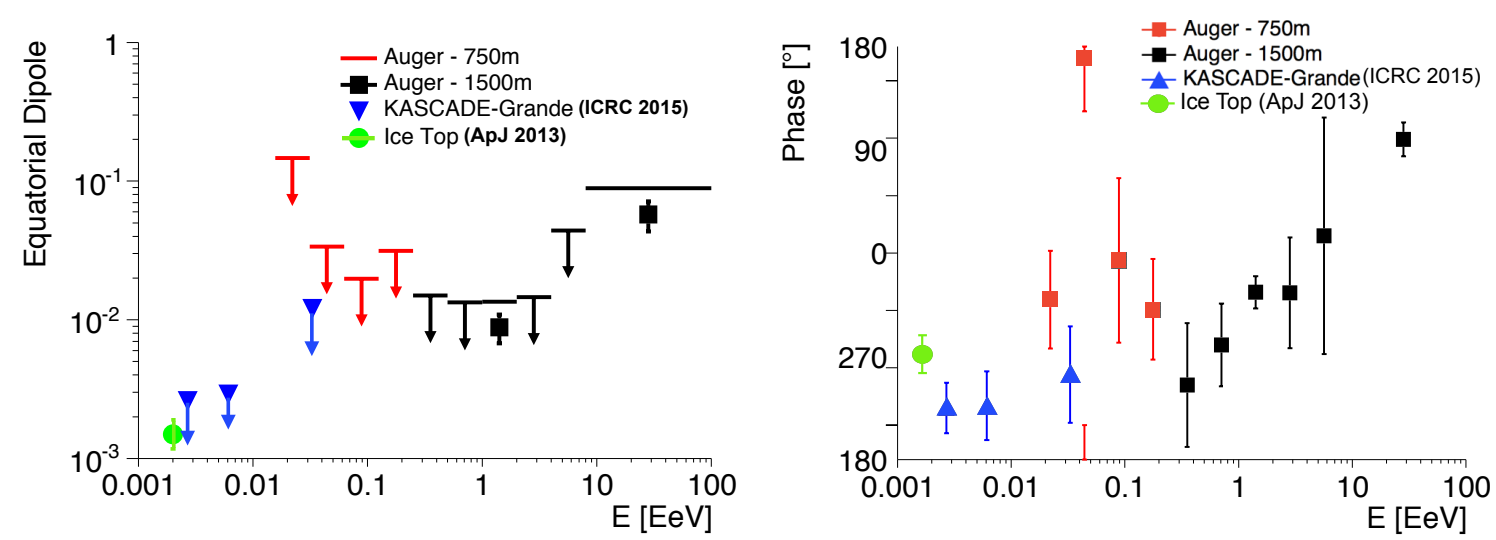

Figure 7: Left: Upper limits to the dipole equatorial component at the 99\% C.L.. Amplitudes are also reported in the two energy bins when the corresponding p-value expected from isotropy is below $10^{-3}$ (see text). Right: Phases of the first harmonic modulation in RA.

trinsic luminosity of the objects. Also, a specific study is done on the distribution of events around the direction of Centaurus A, the closest radio-loud AGN. Out of all the searches, the two largest departures from isotropy (both with post-trial probability $\approx 1.4 \%$ ) are found for cosmic rays with energy above $58 \mathrm{EeV}$. One occurs when looking within $15^{\circ}$ of the direction of Centaurus A. Note that the most significant excess ( $4.3 \sigma$ pre-trial) observed in the blind search corresponds to a region close to the super-Galactic plane and to the direction of Cen A, at a similar energy threshold, $54 \mathrm{EeV}$, and similar angular scale, $12^{\circ}$ (see Fig. 6, left). The other excess is for arrival directions within $18^{\circ}$ of Swift-BAT AGNs closer than $130 \mathrm{Mpc}$ and brighter than $10^{44} \mathrm{erg} / \mathrm{s}$. Overall, none of the analyses shows a statistically significant evidence of anisotropy, including the updated fraction of events correlating with AGNs in the VCV catalog, which does not substantiate the initial evidence of anisotropy at energies larger than $53 \mathrm{EeV}$ [23]. The observed isotropy is amenable to different interpretations. If deflections were small (i.e., if UHECRs were light nuclei), that might indicate a large number of sources. If in turn UHECRs were mostly large- $Z$ nuclei, then the lack of anisotropy might be caused by large deflections. Information on the mass of the primaries at these energies is thus also of relevance in the study of the distribution of the arrival directions.

In the context of testing the distribution of the arrival directions of the highest-energy cosmic rays, it is worth mentioning the result of a full-sky study, conducted in a collaboration among Auger, Telescope Array and IceCube, presented by G. Golup. It consists in the search for correlations between the directions of 318 UHECRs observed by the Auger Observatory (231 events with $E>52 \mathrm{EeV}$ ) and by the Telescope Array (87 events with $E>57 \mathrm{EeV}$ ) and those of very high-energy neutrino candidates detected by IceCube (see Fig. 6, right, for a sky map of the events observed by the three experiments). Although no indications of correlations at discovery level are found, it is interesting to highlight that the smallest post-trial p-values (corresponding to $\sim 3 \sigma$ ) are obtained when considering the correlations between the directions of cascade events observed by IceCube and those of the UHECRs. The excess of correlations, found at angular scales of $\sim 20^{\circ}$, arises mostly from pairs of events in the region of the sky where the Telescope Array has reported an excess of events (so-called hot spot [24]) and in regions close to the super-Galactic plane in 

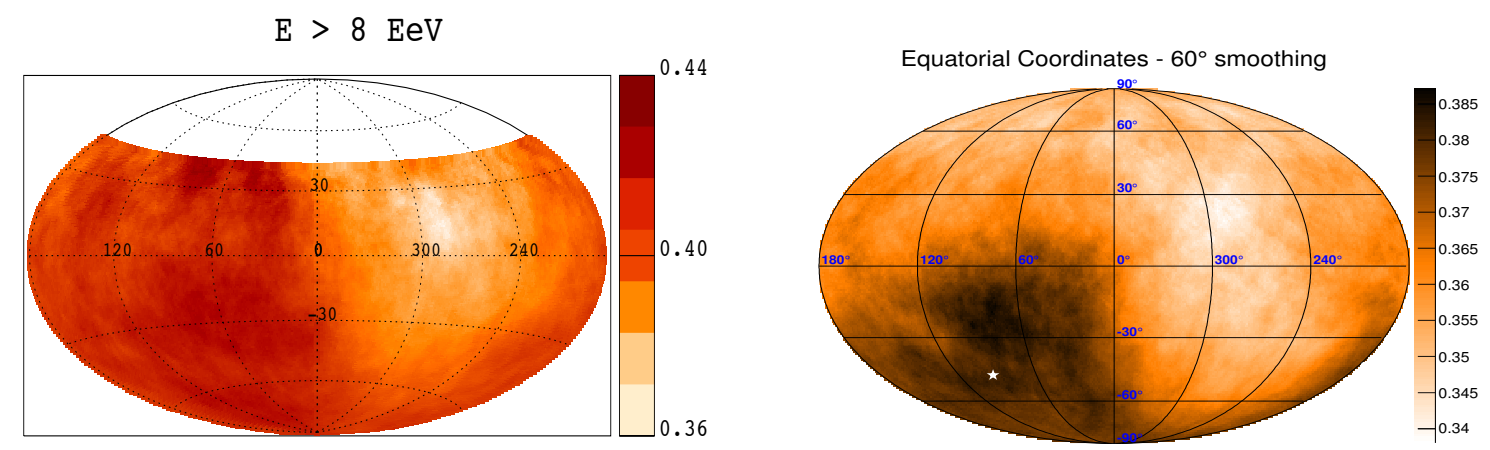

Figure 8: Sky maps in equatorial coordinates of the flux, in $\mathrm{km}^{-2} \mathrm{yr}^{-1} \mathrm{sr}^{-1}$ units, smoothed in angular windows of $45^{\circ}\left(60^{\circ}\right)$ radius, for Auger (Auger and Telescope Array) events with $E>8 \mathrm{EeV}(10 \mathrm{EeV})$, in the left (right) panel.

correspondence with the largest excess observed in Auger data. Notwithstanding the fact that these excesses remain fully compatible with fluctuations of an isotropic distribution, they are warranting a perduring collaboration to monitor them with future data collected by the three experiments.

The last piece of study regarding UHECRs is that of the distribution of arrival directions on large scales, presented by I. Al Samarai. This integrates the previous one, not only in terms of angular scale, but also of inferences, as large-scale anisotropies can be reflective of either a collective motion of cosmic rays (e.g., of their propagation) or of the global distribution of their sources, or of both. As such studies are relevant at all energies, the large-scale analysis has been performed down to the lowest ones accessible by the Observatory, $\sim 10^{16} \mathrm{eV}$. The technique used is that of the harmonic analysis of the counting rate. Above $10^{18} \mathrm{eV}$, where data from the SD-1500 $\mathrm{m}$ are used, this is applied to the rate, corrected for atmospheric and exposure effects, as a function of right ascension (RA). Above $4 \times 10^{18} \mathrm{eV}$ a further harmonic analysis in azimuth is performed, that is sensitive to modulations in declination: the inclusion of horizontal events allows the analysis to be done from $-90^{\circ}$ to $+45^{\circ}$. Below $10^{18} \mathrm{eV}$, where data from both SDs are exploited, the East-West technique [25] is used in turn: the harmonic analysis is applied to the differences in the rate between the Eastern and Western hemispheres, allowing for effects of experimental and atmospheric origin to be removed by the subtraction. Fig. 7 presents a summary of the results of these analyses. As in none of the energy bins are the p-values for the amplitudes of the first harmonic at the level of discovery, the left panel shows the upper limits, at the $99 \%$ C.L., to the equatorial component of the dipole as a function of energy. In the two energy bins where the p-values are $1.5 \times 10^{-4}$ and $6.4 \times 10^{-5}$ (between 1 and $2 \mathrm{EeV}$, and for the integral bin above $8 \mathrm{EeV}$, mean energy $14.5 \mathrm{EeV}$, respectively) amplitudes are also shown. The right panel shows in turn the phases as a function of energy. Interestingly, the phase above $8 \mathrm{EeV}, \simeq 95^{\circ}$ in RA, is roughly the opposite of the one at energies below $1 \mathrm{EeV}$, which is in the general direction of the Galactic centre. Actually, such an evolution of the phases with energy, from $\approx 270^{\circ}$ below $10^{18} \mathrm{eV}$ to $\approx 100^{\circ}$ above $4 \mathrm{EeV}$, had already been pointed out in earlier Auger analyses [26], being the subject of a prescribed test, still in progress. The percent limits to the amplitude of the anisotropy exclude the presence of a large fraction of Galactic protons at EeV energies [27]. Accounting for the inference from $X_{\max }$ data, that protons are in fact abundant at those energies, this might indicate that this component 

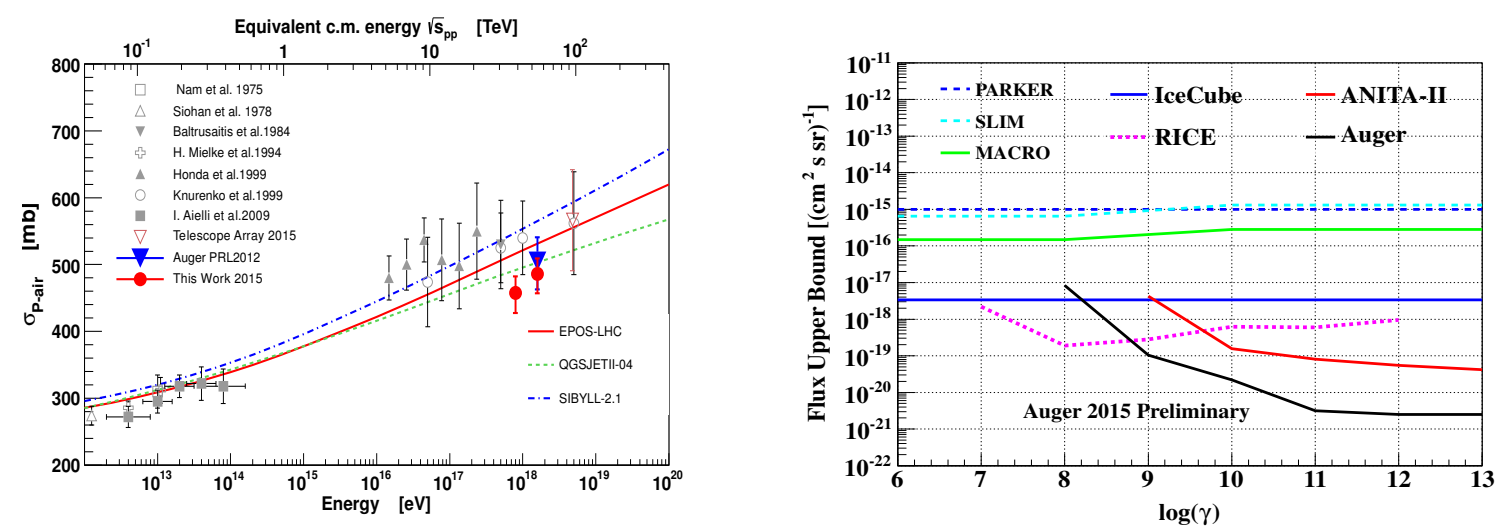

Figure 9: Left: The $\sigma_{\mathrm{p}-\text { air }}$ measurement compared to previous data and model predictions. Right: $90 \%$ C.L. upper limits on the flux of ultra-relativistic IMM, compared to those from other experiments. For detailed references see Ulrich's and Fujii's reports, respectively.

is extragalactic, gradually taking over a Galactic one. The low level of anisotropy would then be the sum of two vectors with opposite directions, naturally reducing the amplitudes. This is an intriguing possibility, to be explored with additional data.

Similarly intriguing is the indication of a departure from isotropy above $8 \mathrm{EeV}$, where the total amplitude of the dipole is found to be $0.073 \pm 0.015$ pointing to $(\alpha, \delta)=\left(95^{\circ} \pm 13^{\circ},-39^{\circ} \pm\right.$ $\left.13^{\circ}\right)$. This finding is robust assuming that the flux of cosmic rays is either purely dipolar or a combination of only dipolar and quadripolar (see also [28]). Assumptions on the shape of the angular distribution can be avoided by analyzing it over the full sky. This has been done through a spherical harmonic analysis of Auger and Telescope Array data [29] and shown in the presentation from O. Deligny. No deviation from isotropy at discovery level is found at any multipoles. The largest deviation from isotropy, with a p-value of $5 \times 10^{-3}$, occurs for the dipolar moment. The amplitude, $0.065 \pm 0.019$, and the direction, $(\alpha, \delta)=\left(93^{\circ} \pm 24^{\circ},-46^{\circ} \pm 18^{\circ}\right)$, are in agreement with those found with Auger-only data. The sky maps of the fluxes in equatorial coordinates, shown in Fig. 8, offer a visualization of the dipolar patterns resulting from the two analyses. Note that I. Valiño has reported the first results on the declination dependence of the energy spectrum. While the comparison of sub-spectra in four declination bands does not show any dependence on $\delta$, the ratio of flux from the southern to the northern sky is compatible with that expected from the dipolar-modulation observed. Such an anisotropy, if confirmed with additional data, might be reflective of the diffusive propagation of UHECRs in the extragalactic magnetic fields and/or of the inhomogenoeus distribution of the sources in our neighborhood. The observed dipolar amplitudes would in particular correspond to expectations in the case of heavier nuclei [30, 31], suggested too by the inferences on mass composition drawn from $X_{\max }$ data.

\section{Beyond ultra-high energy cosmic rays}

Besides studies of UHECRs, the Collaboration has presented a series of contributions that are complementary to those both in terms of reach and of energy. On the one hand, UHECRs reach energies that are far higher than any Earth-based accelerator will ever achieve, so that they represent 

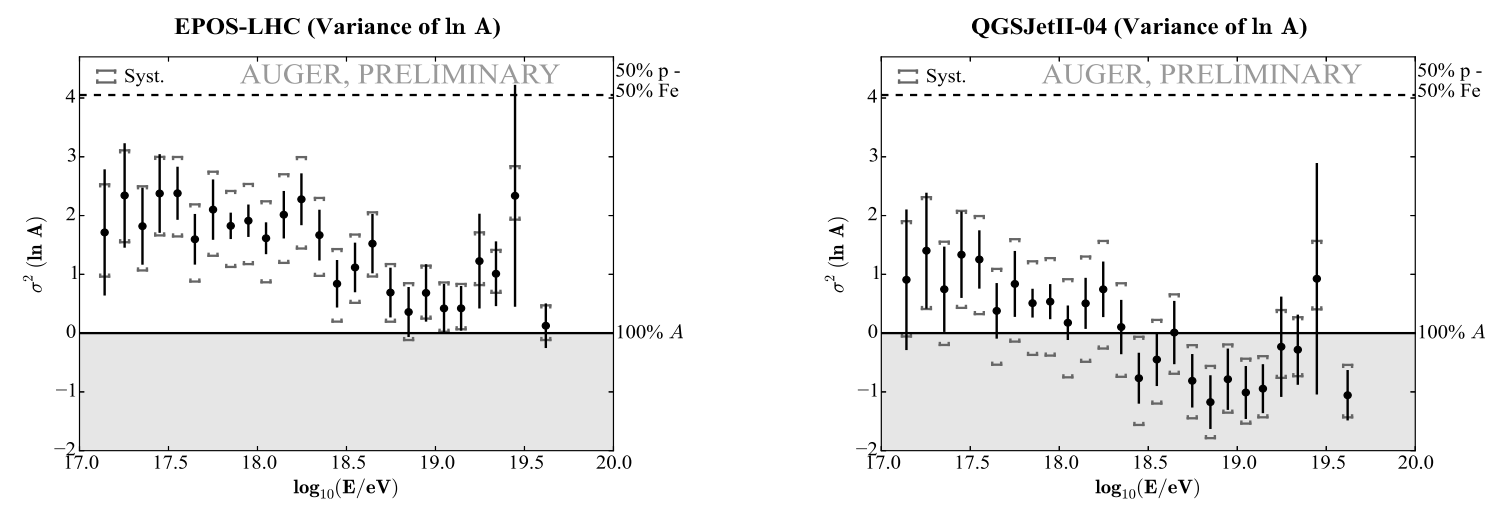

Figure 10: The variance of $\ln A$ inferred from $X_{\max }$ data with EPOS-LHC (left) and QGSJetII-04 (right).

a unique opportunity to access particle interactions in domains, also kinematical, that cannot be explored otherwise. On the other hand, the versatility of the instruments of the Observatory makes the study of the flux of cosmic rays possible down to GeV-TeV energies and places it in the context of the dynamics of the heliosphere and the Sun's activity.

The measurement of the p-air cross-section, illustrated by R. Ulrich, is emblematic of the capability of the Observatory to address particle physics. The work extends, in number of events and energy, the previous one [32], where the measurement was performed in one energy bin only, $10^{18}-10^{18.5} \mathrm{eV}$. The technique is identical, relying on the analysis of the shape of the distribution of the largest measured values of $X_{\max }$. The tail of the $X_{\max }$ distribution is sensitive to the protonair cross-section: it is directly related to the distribution of the first-interaction point of the primary cosmic ray, that in turn is inversely proportional to the p-air cross-section. The observable is $\Lambda_{\eta}$, obtained via the exponential shape of the tail of the $X_{\max }$ distribution, which is a measure of the shower attenuation length in atmosphere. $\eta=20 \%$ is the fraction used of the most deeply penetrating showers, in two energy ranges $\left(10^{17.8}-10^{18} \mathrm{eV}\right.$ and $\left.10^{18}-10^{18.5} \mathrm{eV}\right)$ where the shape of the $X_{\max }$ distribution is compatible with the presence of a substantial fraction of protons and a helium content not larger than $\approx 25 \%$. The measured values of $\Lambda_{\eta}$ are $60.7 \pm 2.1 \pm 1.6 \mathrm{~g} / \mathrm{cm}^{2}$ and $57.4 \pm 1.8 \pm 1.6 \mathrm{~g} / \mathrm{cm}^{2}$ in the two energy bins, respectively, corresponding to centre-of-mass energies of 38.7 and $55.5 \mathrm{TeV}$. As in the previous work, the value of $\sigma_{\mathrm{p}-\text { air }}$ is derived from the comparison of $\Lambda_{\eta}$ with $\Lambda_{\eta}^{\mathrm{MC}}$, calculated from Monte Carlo simulations of air showers. The latter have been updated to include the most recent hadronic models, EPOS-LHC and QGSJETII-04, keeping SIBYLL 2.1 as a reference for pre-LHC models. The two measurements are shown in Fig. 9, left panel, compared to the previous measurement and to model predictions. The data are consistent with a cross-section rising with energy; however, the statistical precision is not yet sufficient to make a statement on the functional form.

Likewise an exemplar of the reach of Auger to fundamental physics, and similarly based on the measurement of the longitudinal shower profile, is the search for ultra-relativistic monopoles, reported by T. Fujii. As the FD observes a huge atmospheric volume, it is in fact a suitable detector to search for signals generated by the electromagnetic interactions in air of magnetic monopoles with large Lorentz factors. As an example, a monopole of intermediate mass (IMM, $M \sim 10^{11}$ $10^{20} \mathrm{eV} / \mathrm{c}^{2}$, Lorentz factor $\gamma \geq 10^{10}$ ) with $\gamma=10^{11}$ loses $\approx 400 \mathrm{PeV} /\left(\mathrm{g} / \mathrm{cm}^{2}\right)$ which corresponds to 

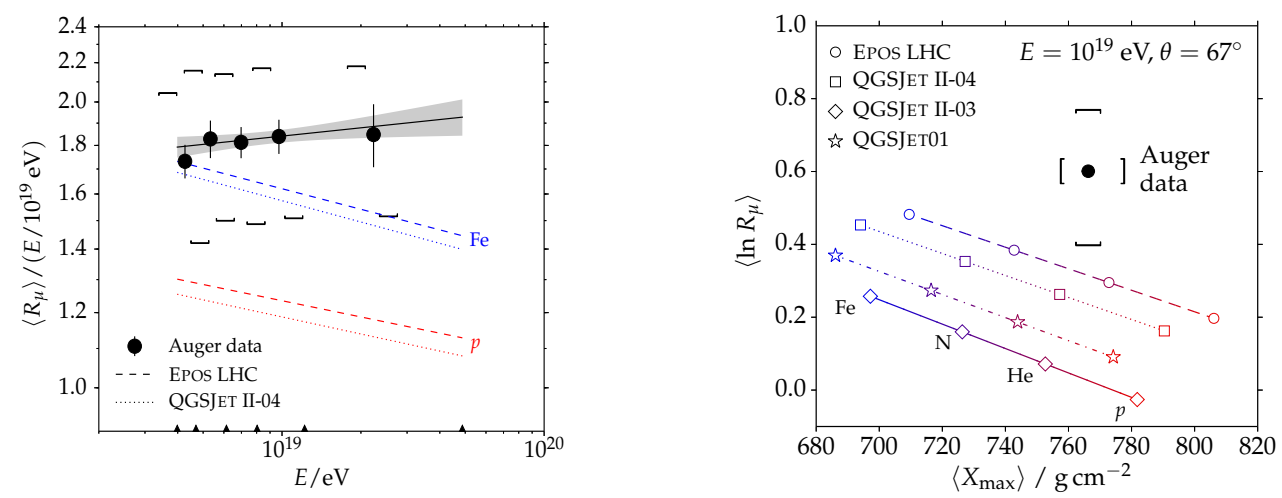

Figure 11: Left: $\left\langle R_{\mu}\right\rangle$ as a function of primary energy, compared to air-shower simulations. Right: $\left\langle\ln R_{\mu}\right\rangle$ as a function of $\left\langle X_{\max }\right\rangle$. Representative primary masses are indicated by open symbols: the predictions for proton and iron showers are directly taken from simulations. Values for intermediate masses are computed with the Heitler model.

$\approx 10^{20.5} \mathrm{eV}$ when integrated over an atmospheric depth of $\approx 1000 \mathrm{~g} / \mathrm{cm}^{2}$. This energy is dissipated by monopoles through production of secondary showers along its path. When compared with a standard UHECR proton shower of energy $10^{20} \mathrm{eV}$, the IMM shower presents a much larger energy deposit and deeper development, due to the superposition of many showers uniformly produced along the monopole's path in the atmosphere. Based on these features, events with a very large slant depth at the upper FOV boundary of the FD, in conjunction with a very large deposited energy, are searched for as monopole candidates. The selection criteria are tuned using Monte Carlo simulations and complemented by $10 \%$ of data. No candidates are found when unblinding the rest of the 10-yr data sample. The corresponding upper limits for different Lorentz factors are shown in Fig. 9, right, together with results from previous experiments. The Auger limit turns out to be the best one for ultra-relativistic IMM for $\gamma \geq 10^{9}$, with a factor of ten improvement with respect to previous experiments for $\gamma \geq 10^{10}$.

The reach of the data of the Observatory to particle interactions is also evinced from the ensemble of contributions that show the significance of mass-related observables with respect to the validity of hadronic interaction models. As mass intepretation relies on the comparison of observations with predictions of simulations using high-energy interaction models, mass-sensitive observables are in fact model-sensitive too. Different observables, accessible either to FD (hence mostly sensitive to the EAS electromagnetic component) or to SD (sensitive to the muonic and electromagnetic components) play this double role.

The first clue to an inadequacy of current models in covering the complexity of UHE showers is indeed provided by $X_{\max }$ data, when interpreting them in terms of $\ln A$ via different models. As first shown in [33], and updated in the contribution of A. Porcelli, the trends of both $\ln A$ moments as a function of energy are consistent among models, leading to consistent inferences on the evolution of the mass composition. However, and as shown in Fig. 10, the values of $\sigma^{2}(\ln \mathrm{A})$ turn out to be unphysical at the highest energies when the conversion is made via QGSJETII-04. These values occur for energies where the intrinsic shower fluctuations predicted by this model for the $\langle\ln A\rangle$ exceed the measured ones. The systematic uncertainties of the analysis do not allow the rejection of any model, but the clue found brings up the interest of probing hadronic models by measuring 

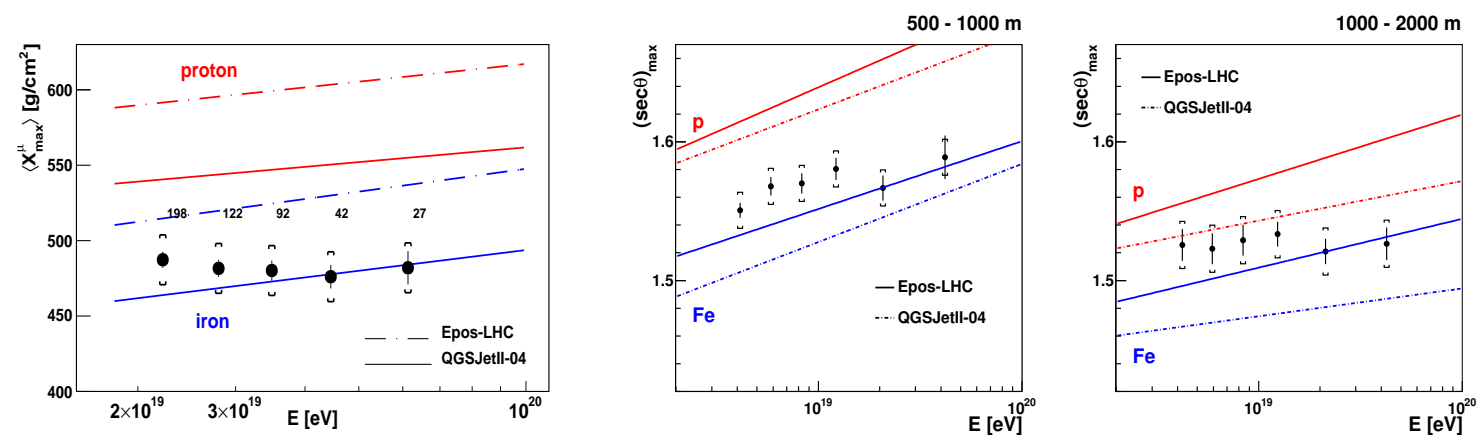

Figure 12: Left: Evolution of $\left\langle X_{\max }^{\mu}\right\rangle$ with energy. Centre and right: Evolution of $(\sec \theta)_{\max }$ with energy in two different core-distance ranges. In all figures brackets represent the systematic uncertainty.

mass-sensitive observables accessing other EAS components. The muonic component is one of those, and it is at the heart of two studies presented by L. Collica, both based on the analysis of horizontal showers. These are characterized by the dominance of muons, as the electromagnetic component is mostly absorbed in the large atmospheric depth crossed.

One study concentrates on the number of muons, which is estimated through $N_{19}$, the scale factor that relates the observed muon densities to the average muon density profile in simulated $10 \mathrm{EeV}$ proton showers [34]. As seen in Section 2, it is well-correlated with the primary energy: hybrid horizontal events not only make their energy calibration possible, but they also allow the study of the evolution of the number of muons as a function of energy (see also [35]). Fig. 11, left, shows the average values of the unbiased $N_{19}\left(R_{\mu}\right.$, corrected through simulations), divided by energy, obtained with 174 hybrid events with energy above $4 \times 10^{18} \mathrm{eV}$. Data are compared to simulations of proton and iron showers, performed with QGSJETII-04 and EPOS-LHC. Such a comparison allows for two different kinds of considerations. On the one hand, the well-separated predictions for protons and iron nuclei confirm that $\left\langle R_{\mu}\right\rangle$ is an efficacious composition estimator. The observed energy dependence of $\left\langle R_{\mu}\right\rangle$ with respect to that of either species suggests a transition from lighter to heavier elements, consistent with what is seen in the evolution of $\left\langle X_{\max }\right\rangle$ (see Fig. 3). On the other hand, a second clue on the shortcomings of the models shows up, as $\left\langle R_{\mu}\right\rangle$ turns out to be higher than that expected, consistently for the two models, in pure iron showers. This in turn is in contrast with $X_{\max }$ data: the tension between the two measurements can be better appreciated in Fig. 11, right. Here $\left\langle\ln R_{\mu}\right\rangle$, the mean logarithmic muon content measured at $10^{19} \mathrm{eV}$, is compared to expectations based on $\langle\ln A\rangle$ as inferred by $X_{\max }$ data, for different hadronic models and primary masses. The second clue is here accentuated, as none of the models predicts the observation well, with EPOS-LHC predictions being in this case closest to the data. The mean number of muons is predicted by simulations at a deficit of $30 \%$ to $80_{-20}^{+17}$ (syst.) $\%$ at $10^{19} \mathrm{eV}$, depending on the model.

The second study investigates the muon production depth (MPD). This is assessed in SD events through the measurement of the time delay of muons with respect to the shower front, this being reflective of their production height. The measurement of the MPD (see also [36]) is performed in stations far from the shower core in $\sim 500$ events with $55^{\circ}<\theta<65^{\circ}$ and with $E>2 \times 10^{19} \mathrm{eV}$. MPD distributions are then derived at different energies, each fitted with a Gaisser-Hillas function from which the muonic shower maximum, $X_{\max }^{\mu}$, is determined. The evolution of $\left\langle X_{\max }^{\mu}\right\rangle$ with energy is shown in Fig. 12, left, for data and simulations based on EPOS-LHC and QGSJETII-04. 

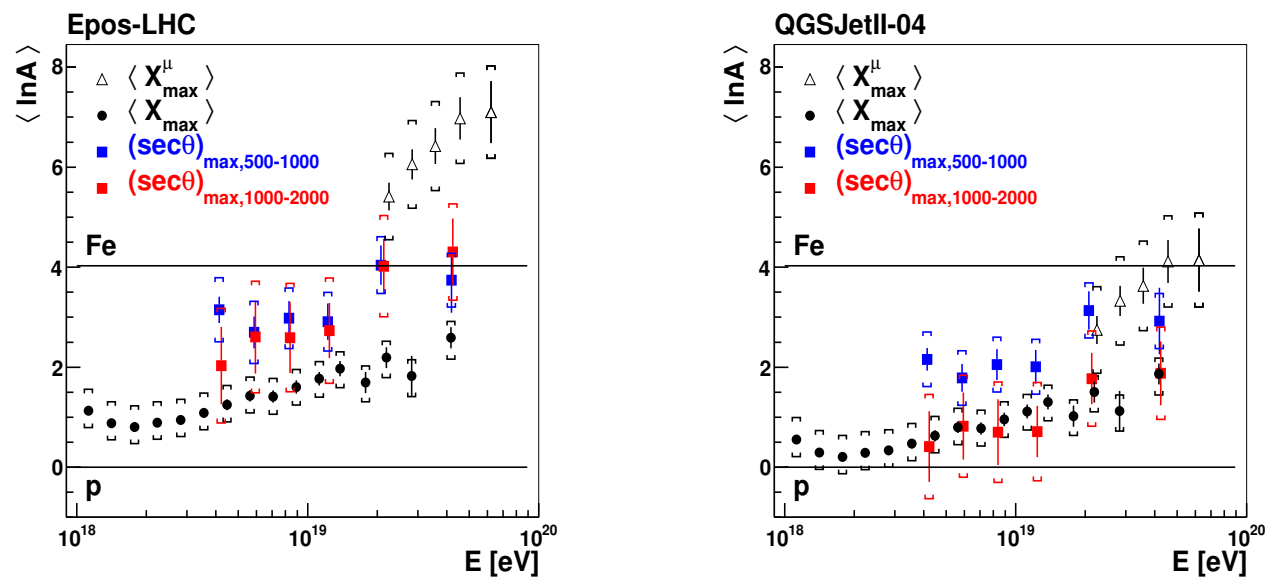

Figure 13: $\langle\ln \mathrm{A}\rangle$ as a function of energy as predicted by EPOS-LHC (left) and QGSJetII-04 (right), from $\left\langle X_{\max }\right\rangle$ (dots), $\left\langle X_{\max }^{\mu}\right\rangle$ (triangles) and $(\sec \theta)_{\max }$ data (squares).

This figure also allows for two lines of thought. Predictions for proton and iron primaries in individual models are well-separated, as expected for an observable sensitive to the EAS longitudinal development. The trend of data with energy, flatter than that of either species predictions by either model, disfavors pure protons or iron primaries, in agreement with $X_{\max }$ measurements. On the other hand, a further clue to the shortcoming of models appears, in the form of a considerable difference in the absolute predicted value of $\left\langle X_{\max }^{\mu}\right\rangle$. Predictions of QGSJETII-04 better represent observations, as they bracket them; in turn, data are far underneath the predictions of EPOS-LHC, being well-below even those for iron primaries. This is more evident when converting $\left\langle X_{\max }^{\mu}\right\rangle$ to $\langle\ln \mathrm{A}\rangle$ using the two models. As shown in Fig. 13, the outcome of the conversion for EPOS-LHC (left panel, triangles) is that primaries are heavier than iron: this is incompatible at a level of at least $6 \sigma$ with the $\langle\ln A\rangle$ inferred from $\left\langle X_{\max }\right\rangle$ (dots). With QGSJETII-04 (right panel), the $\langle\ln A\rangle$ values inferred by the two measurements (triangles and dots) are in turn compatible within $1.5 \sigma$.

The azimuthal asymmetry in the risetime of signals observed in the WCDs, presented by I. Minaya, completes the set of the studied mass/model-sensitive observables. Not present in vertical showers, the asymmetry increases with the zenith angle $\theta$, reaching a maximum. The relative proportion of the electromagnetic and muonic shower components plays a role in such evolution, correlated with the longitudinal shower development. The angle of maximal asymmetry is sensitive to the primary mass [37]. The magnitude of the asymmetry, as well as its evolution as a function of $\theta$, depends also on the core distance, due to geometrical effects and to the electromagnetic/muonic ratio varying with distance. The analysis is thus carried out in two different ranges, i.e., $500-1000$ $\mathrm{m}$ and $1000-2000 \mathrm{~m}$, using $\sim 55000$ events with $E>3 \times 10^{18} \mathrm{eV}$ and $\theta>30^{\circ}$. The risetime is evaluated for each WCD; the azimuthal asymmetry is then computed by including all stations at a given $E$ and $\theta$, for each distance range. From the distribution of the asymmetry in $\theta$, the angle at which the asymmetry is maximal, $(\sec \theta)_{\max }$, is determined as a function of energy. The evolution of $(\sec \theta)_{\max }$ as a function of energy in the two distance ranges is shown in the central and right panels of Fig. 12, both for data and for simulations based on EPOS-LHC and QGSJETII-04. Also for this observable, predictions for proton and iron primaries are well-separated in both models 
and at both distances, demonstrating the sensitivity to mass of $(\sec \theta)_{\max }$. The evolution of data with energy, flatter than that predicted by either model, species, and distance, hints at a transition from lighter to heavier composition. Composition inferences from this observable are however particularly complicated as the predictions of the models are at variance not only between them but even in different distance ranges. This can be better grasped by looking at the values of $\langle\ln A\rangle$ inferred from $(\sec \theta)_{\max }$, shown as squares in Fig. 13. The values of $\langle\ln A\rangle$ derived from EPOS-LHC (left) are consistent, within uncertainties, in the two distance ranges, but they are only marginally compatible with those inferred from $\left\langle X_{\max }\right\rangle$ and $\left\langle X_{\max }^{\mu}\right\rangle$, also shown in the figure. The consistency in distance range is in turn less evident when the conversion is made using QGSJETII-04 (right), hindering any reasonable comparison with the other two measurements.

The complex picture of hadronic models deriving from the series of analyses presented allows nevertheless for a few experimental considerations. The studies of different EAS characteristics linked to different components, as allowed by the hybrid nature of the Observatory, provide constraints on interactions models stronger than those obtained by means of single observables or single detectors. While no model seems to provide a completely satisfactory description of the wide variety of measurements, the latter provide unique feedbacks on these models, at energies that are not otherwise accessible. Additional composition-sensitive information is awaited to further help in reducing systematic uncertainties related to modeling hadronic showers: the Collaboration is already working on complementary observables, such as the average longitudinal profile (presented by F. Diogo) or the muon content in hybrid events [38]. On the other hand, and in spite of the shortcomings highlighted, it is undoubtable that the quality and predictive power of air-shower simulations have dramatically improved in the last two decades. The consistency of the predictions for $\left\langle X_{\max }\right\rangle$ (see Fig. 3) is remarkable, leading to rather robust interpretations in terms of mass evolution with energy. The fact that the interpretations of all SD-based observables overall indicate a similar trend is encouraging us to pursue the measurement of the mass composition up to the highest accessible energies.

To close this section, the last highlight is reserved for an analysis that turns to dramatically different energies and inferences. J. Masias's study exploits the rate of signals recorded by the SD at a very low threshold, normally employed for monitoring and calibration. The range of deposited energy in the WCD, between $\sim 15 \mathrm{MeV}$ and $\sim 1 \mathrm{GeV}$, corresponds to primary energies between $\mathrm{O}(10$ $\mathrm{GeV})$ and $\mathrm{O}(\mathrm{TeV})$ [39]. At these energies the cosmic-ray flux is heavily modulated by solar effects. The data cover 8 years across Solar cycles 23 and 24 . The long-term behavior

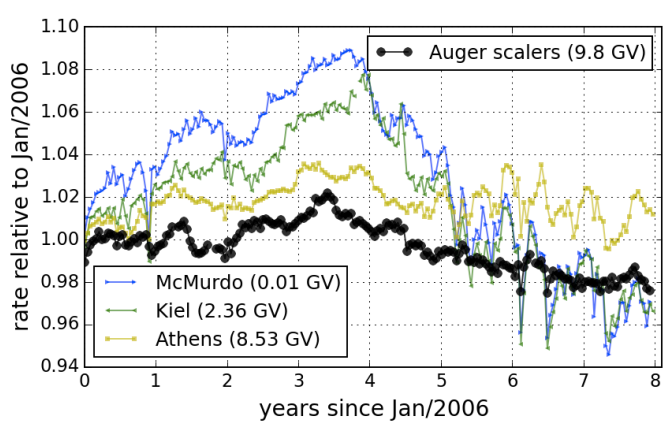

Figure 14: Counting rate over the years as obtained by the scaler trigger, compared with different neutron monitors (the inset shows geomagnetic rigidity cutoff). of the counting rates is studied in two different bins of deposited energy: 60-120 MeV and 200-280 MeV. Rates are corrected for long-term variations of the response of the WCD and for atmospheric effects. Fig. 14 shows the rates over the years as observed in the lower-energy bin (primary energy $\sim 90 \mathrm{GeV}$ ). They are compared 
with those obtained by data from different neutron monitors (McMurdo, Kiel, and Athens), which have different rigidity cutoffs depending on their location. A global maximum near the middle of 2009 is clearly visible in Auger data, in agreement with the observation of neutron monitors: the maximum is associated with the minimum of solar activity, toward the beginning of the Solar cycle 24. The observed amplitude of the modulation is the lowest one, as the geomagnetic rigidity cutoff at the Auger site is the largest one. Coherently, the amplitude turns to be of lower amplitude in the higher-energy bin, as shown by Masias. It is worth noting that Auger data, in both bins, correspond to higher energies than observed in neutron monitors, which moreover cannot distinguish between different energies. Furthermore, Auger data provide the flux of cosmic rays with the highest statistical significance due to its huge total collecting area. These observations thus complement and supplement those of traditional instruments in understanding the effects of different processes affecting the cosmic-ray transport in the heliosphere.

\section{Conclusions and perspectives}

At the time of the conception of the Pierre Auger Observatory, nearly 25 years ago, the knowledge of cosmic rays at the highest energies was very limited. Their flux above $10 \mathrm{EeV}$ was unresolved, prompting exotic models for their origin and propagation; their chemical composition was completely unexplored; the distribution of their arrival directions, due to the meager numbers, was a harbinger of contradictory interpretations in terms of their anisotropy. The huge collecting area and the hybrid detection strategy of the Observatory have yielded dramatic advances in the measurements of UHECRs, as planned. The latter have in turn largely affected the traditional intuition on UHECRs. On the one hand, the clearly observed suppression in the flux, starting at around $40 \mathrm{EeV}$, is evocative of the GZK cutoff. However, when compared with the inferred trend of the mass composition towards such an energy (i.e., a gradual shift towards heavier primaries), it seems to support a cutoff intrinsically due to the sources as well as to the propagation of UHECRs. On the other hand, the very stringent limits on the flux of UHE photons exclude most of the so-called top-down models for the UHECR production in favor of their acceleration in astrophysical source models. However, no evidence of anisotropy or of association with astrophysical sources has been found when looking at the small- and intermediate-scale distribution of the arrival directions of hundreds of UHECRs above $40 \mathrm{EeV}$. The high degree of isotropy challenges the common wisdom that UHECRs are mostly protons, as does the indication of a dipole in the distribution of cosmic rays above $\sim 10 \mathrm{EeV}$.

The mass composition of UHECRs into the suppression region remains unexplored. The FD, on whose data the mass interpretation is based, loses statistical power above $40 \mathrm{EeV}$, due to its limited duty cycle. The refined and creative analyses of data from the $100 \%$ duty cycle SD have provided a variety of mass-sensitive observables, but they require very severe selections that largely reduce the number of exploitable events. Still, as for any of the spectral features observed in the cosmic-ray spectrum, information on the primary mass is a necessity to understand the origin of the flux suppression. Also, mass-dependent studies of the arrival directions of UHECRs might be relevant to understand the reasons for the lack of small-scale anisotropy at the highest energies. It follows from these considerations that equipping the SD to assess mass composition at the highest energies is the natural evolution of the Observatory in furtherance of its mission. As presented by 
R. Engel, the Collaboration has devised an upgrade of the SD that allows the determination of the EAS muonic component, the principal mass-sensitive observable for EAS arrays. In keeping with the hybrid detection strategy, the upgrade, dubbed AugerPrime, foresees the installation of a plane of $4 \mathrm{~m}^{2}$ plastic scintillators above each WCD. The sampling of EAS particles with two detectors with different and complementary responses to the muonic and electromagnetic components will allow the separate reconstruction of both. The muonic component, station by station, will be derived by subtracting the signal observed in the scintillator from that seen in the WCD. The signal at an optimal distance, determined by a fit to the lateral distribution of the so-obtained signals, will be used as mass estimator. The number of muons will also be assessed by means of a multi-variate analysis of the signals from the complementary detectors based on the concept of EAS universality [40]: not only will the magnitude of the signals be exploited, but also their temporal structure. The upgrade of the SD will also be the occasion to modernize its electronics: faster FADCs (120 $\mathrm{MHz}$ sampling compared to the current $40 \mathrm{MHz}$ ) and an increased dynamic range are among the improvements foreseen.

The operation of such an upgraded array, anticipated between 2018 and 2024, will allow the study of mass composition in the region of the flux suppression with as many events again as collected so far. While the main focus of the upgrade is on the SD and on the highest-energy end of the spectrum, i.e., above $10 \mathrm{EeV}$, neither the FD nor the lower-energy range will be disregarded. The operation mode of the FD will be changed to extend measurements into night periods with a higher light background: an increase of $\sim 50 \%$ in the on-time is projected. All WCDs in the SD-750 m will be equipped with scintillators as well. In parallel, the deployment of the buried scintillators of AMIGA (whose engineering array of 7 units is now in operation, as detailed by B. Wundheiler) will be completed. Besides addressing the subject of mass composition at $0.1 \mathrm{EeV}$, muon measurements by AMIGA will also serve as a verification of the methods envisioned to extract the muonic signals from the combination of scintillators and WCDs.

\section{References}

[1] The Pierre Auger Collaboration, Nucl. Instrum. Meth. A 798 (2015) 172.

[2] K. Greisen, Phys. Rev. Lett. 16 (1966) 748; G. T. Zatsepin and V. A. Kuz'min, JETP Lett. 4 (1966) 78 [Pisma Zh. Eksp. Teor. Fiz. 4 (1966) 114].

[3] M. Boratav for the Pierre Auger Collaboration, in Proc. $25^{\text {th }}$ ICRC, Durban, 5 (1997) 205.

[4] The Pierre Auger Collaboration, arXiv:1509.03732.

[5] The Telescope Array and Pierre Auger Collaborations, arXiv:1511.02103.

[6] The IceCube, Auger and Telescope Array, arXiv:1511.02109.

[7] V. Verzi, for the Pierre Auger Collaboration, in Proc. $33^{r d}$ ICRC, Rio de Janeiro (2013), arXiv:1307.5059.

[8] The Pierre Auger Collaboration, Phys. Rev. Lett. 101 (2008) 061101; Phys. Lett. B 685 (2010) 239.

[9] The Pierre Auger Collaboration, Phys. Rev. Lett. 104 (2010) 091101.

[10] The Pierre Auger Collaboration, Phys. Rev. D 90 (2014) 122005.

[11] S. Ostapchenko, Phys. Rev. D 83 (2011) 014018. 
[12] T. Pierog and K. Werner, Phys. Rev. Lett. 101 (2008) 171101; T. Pierog et al., arXiv:1306.0121.

[13] E. J. Ahn, R. Engel, T. Gaisser, P. Lipari, and T. Stanev, Phys. Rev. D 80 (2009) 094003.

[14] The Pierre Auger Collaboration, Phys. Rev. D 90 (2014) 122006.

[15] V. Berezinsky, A.Z. Gazizov and S.I. Grigorieva, Phys. Lett. B 612 (2005) 147; Phys. Rev. D 74 (2006) 043005.

[16] P. Younk and M. Risse, Astrop. Phys. 35 (2012) 807.

[17] R. Aloisio et al., JCAP 1210 (2012) 007.

[18] J. Bahcall and E. Waxman, Phys. Rev. D 64 (2001) 023002.

[19] The Pierre Auger Collaboration, ApJ 804 (2015) 15.

[20] J.P. Huchra et al., ApJS 199 (2012) 26.

[21] W.H. Baumgartner et al., ApJS 207 (2013) 19.

[22] S. Van Velzen, et al., A\&A 544 A18.

[23] The Pierre Auger Collaboration, Science 318 (2007) 938; Astrop. Phys. 34 (2010) 314.

[24] The Telescope Array Collaboration, ApJ 790 (2014) L21.

[25] R. Bonino et al., ApJ 738 (2011) 67

[26] I. Sidelnik, for the Pierre Auger Collaboration, Proc. 33rd ICRC, Rio de Janeiro, Brazil (2013) [arXiv:1307.5059].

[27] The Pierre Auger Collaboration, ApJS 203 (2012) 34.

[28] The Pierre Auger Collaboration, ApJ 802 (2015) 111.

[29] The Pierre Auger and Telescope Array Collaborations, ApJ 794 (2014) 172.

[30] D. Harari, S. Mollerach, E. Roulet, Phys.Rev. D 92 (2015) 063014.

[31] P. Tinyakov, F. Urban, J. Exp. Theor. Phys. 120 (2015) 533.

[32] The Pierre Auger Collaboration, Phys. Rev. Lett. 109 (2012) 3062002.

[33] The Pierre Auger Collaboration, JCAP 02 (2013) 026.

[34] The Pierre Auger Collaboration, JCAP 08 (2014) 019.

[35] The Pierre Auger Collaboration, Phys. Rev. D 91 (2015) 032003; Erratum: Phys. Rev. D 91 (2015) 059901.

[36] The Pierre Auger Collaboration, Phys. Rev. D 90 (2014) 012012; Erratum: Phys.Rev. D 90 (2014) 039904 and Phys.Rev. D 92 (2015) 019903

[37] M. Dova et al., Astrop. Phys. 31 (2009) 312.

[38] G.R. Farrar, for the Pierre Auger Collaboration, Proc. 33rd ICRC, Rio de Janeiro, Brazil (2013), [arXiv:1307.5059].

[39] S. Dasso and H. Asorey, Adv. in Space Res. 49 (2012) 1563

[40] M. Ave et al., Proc. 31 st ICRC, Beijing, China, 2 (2011) 178 
List of ICRC 2015 contributions authored by the Pierre Auger Collaboration

\begin{tabular}{|c|c|c|c|}
\hline Presenter & Title & PoS & Ref. \\
\hline I. Valiño & $\begin{array}{l}\text { The flux of ultra-high energy cosmic rays after ten years of operation of the } \\
\text { Pierre Auger Observatory }\end{array}$ & PoS (ICRC2015) 271 & [4] \\
\hline J. Aublin & $\begin{array}{l}\text { Arrival directions of the highest-energy cosmic rays detected with the Pierre } \\
\text { Auger Observatory }\end{array}$ & PoS (ICRC2015) 310 & [4] \\
\hline I. Al Samarai & $\begin{array}{l}\text { Indications of anisotropy at large angular scales in the arrival directions of } \\
\text { cosmic rays detected at the Pierre Auger Observatory }\end{array}$ & PoS (ICRC2015) 372 & [4] \\
\hline T. Winchen & $\begin{array}{l}\text { Search for energy dependent patterns in the arrival directions of cosmic rays } \\
\text { at the Pierre Auger Observatory }\end{array}$ & PoS (ICRC2015) 294 & [4] \\
\hline A. Porcelli & $\begin{array}{l}\text { Measurements of } X_{\max } \text { above } 10^{17} \mathrm{eV} \text { with the fluorescence detector of the } \\
\text { Pierre Auger Observatory }\end{array}$ & PoS (ICRC2015) 420 & [4] \\
\hline A. Yushkov & $\begin{array}{l}\text { Composition at the "ankle" measured by the Pierre Auger Observatory: pure } \\
\text { or mixed? }\end{array}$ & PoS (ICRC2015) 335 & [4] \\
\hline T. Fujii & $\begin{array}{l}\text { Search for ultra-relativistic magnetic monopoles with the Pierre Auger Ob- } \\
\text { servatory }\end{array}$ & PoS (ICRC2015) 319 & [4] \\
\hline C. Bleve & $\begin{array}{l}\text { Updates on the neutrino and photon limits from the Pierre Auger Observa- } \\
\text { tory }\end{array}$ & PoS (ICRC2015) 1103 & [4] \\
\hline I. Minaya & $\begin{array}{l}\text { Time asymmetries in the Surface Detector signals of the Pierre Auger Ob- } \\
\text { servatory }\end{array}$ & PoS (ICRC2015) 405 & [4] \\
\hline L. Collica & $\begin{array}{l}\text { Measurement of the muon content in air showers at the Pierre Auger Obser- } \\
\text { vatory }\end{array}$ & PoS (ICRC2015) 336 & [4] \\
\hline R. Ulrich & $\begin{array}{l}\text { Extension of the measurement of the proton-air cross section with the Pierre } \\
\text { Auger Observatory }\end{array}$ & PoS (ICRC2015) 401 & [4] \\
\hline F. Diogo & $\begin{array}{l}\text { Measurement of the average electromagnetic longitudinal shower profile at } \\
\text { the Pierre Auger Observatory }\end{array}$ & PoS (ICRC2015) 413 & [4] \\
\hline A. di Matteo & $\begin{array}{l}\text { Combined fit of spectrum and composition data as measured by the Pierre } \\
\text { Auger Observatory }\end{array}$ & PoS (ICRC2015) 249 & [4] \\
\hline J. Masias-Meza & $\begin{array}{l}\text { Solar cycle modulation of cosmic rays observed with the low energy modes } \\
\text { of the Pierre Auger Observatory }\end{array}$ & PoS (ICRC2015) 074 & [4] \\
\hline J. Rautenberg & Lightning detection at the Pierre Auger Observatory & PoS (ICRC2015) 678 & [4] \\
\hline C. Medina & $\begin{array}{l}\text { Atmospheric monitoring at the Pierre Auger Observatory using the upgraded } \\
\text { Central Laser Facility }\end{array}$ & PoS (ICRC2015) 624 & [4] \\
\hline R. Engel & Upgrade of the Pierre Auger Observatory & PoS (ICRC2015) 686 & [4] \\
\hline B. Wundheiler & $\begin{array}{l}\text { The AMIGA muon counters of the Pierre Auger Observatory: performance } \\
\text { and studies of the lateral distribution function }\end{array}$ & PoS (ICRC2015) 324 & [4] \\
\hline C. Glaser & $\begin{array}{l}\text { The energy content of extensive air showers in the radio frequency range of } \\
30-80 \mathrm{MHz}\end{array}$ & PoS (ICRC2015) 364 & [4] \\
\hline P. Assis & $\begin{array}{l}\text { Measurement of the water-Cherenkov detector response to inclined muons } \\
\text { using an RPC hodoscope }\end{array}$ & PoS (ICRC2015) 620 & [4] \\
\hline G. Salina & $\begin{array}{l}\text { Automated procedures for the Fluorescence Detector calibration at the } \\
\text { Pierre Auger Observatory }\end{array}$ & PoS (ICRC2015) 594 & [4] \\
\hline J. Schulz & Status and prospects of the Auger Engineering Radio Array & PoS (ICRC2015) 615 & [4] \\
\hline A. Haungs & $\begin{array}{l}\text { AugerNext: R\&D studies at the Pierre Auger Observatory for a next gener- } \\
\text { ation ground-based ultra-high energy cosmic ray experiment }\end{array}$ & PoS (ICRC2015) 593 & [4] \\
\hline C. Timmermans & Education, outreach and public relations of the Pierre Auger Observatory & PoS (ICRC2015) 587 & [4] \\
\hline M. Unger & $\begin{array}{l}\text { Report of the working group on the composition of ultra-high energy cosmic } \\
\text { rays }\end{array}$ & PoS (ICRC2015) 307 & [5] \\
\hline O. Deligny & $\begin{array}{l}\text { Large-scale distribution of arrival directions of cosmic rays detected at the } \\
\text { Pierre Auger Observatory and the Telescope Array }\end{array}$ & PoS (ICRC2015) 395 & {$[5]$} \\
\hline R. Takeishi & $\begin{array}{l}\text { Initial results of a direct comparison between the Surface Detectors of the } \\
\text { Pierre Auger Observatory and of the Telescope Array }\end{array}$ & PoS (ICRC2015) 393 & [5] \\
\hline G. Golup & $\begin{array}{l}\text { Correlation between the UHECRs measured by the Pierre Auger Observa- } \\
\text { tory and Telescope Array and neutrino candidate events from IceCube }\end{array}$ & PoS (ICRC2015) 1082 & [6] \\
\hline
\end{tabular}

